\title{
BMJ Open To what extent is the elevated risk of psychological distress in people with diabetes accounted for by physical disability? Findings from a large population-based study
}

\author{
Elza Elmira, ${ }^{1}$ Emily Banks, ${ }^{2}$ Grace Joshy ${ }^{1}$
}

To cite: Elmira E, Banks E, Joshy $G$. To what extent is the elevated risk of psychological distress in people with diabetes accounted for by physical disability? Findings from a large populationbased study. BMJ Open 2018;8:e022767. doi:10.1136/ bmjopen-2018-022767

- Prepublication history for this paper is available online. To view these files, please visit the journal online (http://dx.doi org/10.1136/bmjopen-2018022767).

Received 5 March 2018 Revised 20 July 2018 Accepted 6 August 2018

Check for updates

(c) Author(s) (or their employer(s)) 2018. Re-use permitted under CC BY-NC. No commercial re-use. See rights and permissions. Published by BMJ.

${ }^{1}$ National Centre for Epidemiology and Population Health, Research School of Population Health, Australian National University, Canberra, Australian Capital Territory, Australia

${ }^{2}$ The Sax Institute, The Sax Institute, Sydney, New South Wales, Australia

Correspondence to Dr Grace Joshy; grace.joshy@anu.edu.au

\section{ABSTRACT}

Objectives People with diabetes experience an elevated risk of psychological distress compared with people without diabetes. It is unclear how much of this elevated risk is attributable to the greater levels of physical disability in people with diabetes, and how this risk varies according to sociodemographic and healthbehavioural characteristics. This study quantified levels of psychological distress in people with and without diabetes, considering these factors.

Design Cross-sectional analysis of questionnaire data. Setting Men and women aged $\geq 45$ years, in the 45 and Up Study, from New South Wales, Australia.

Participants 236441 people who completed the baseline postal questionnaire (distributed from 1 January 2006-31 December 2008), with valid data for diabetes status and psychological distress.

Primary outcome measures High psychological distress (Kessler-10 >22). Modified Poisson regression with robust error variance was used to estimate prevalence ratios (PRs), comparing prevalence of high psychological distress among those with and without diabetes and across physical functional limitation (PFL) levels, adjusting for potential confounders.

Results Overall, 8.4\% (19 803/236 441) of participants reported diabetes. $11.8 \%$ (2339) of individuals with diabetes and $7.2 \%$ (15 664) without diabetes had high psychological distress: age-adjusted and sexadjusted $\mathrm{PR}=1.89$ (95\% $\mathrm{Cl} 1.81$ to 1.97$)$, becoming 1.58 (1.52 to 1.65) and 1.22 (1.17 to 1.27) following additional adjustment for sociodemographic factors, health behaviours and additionally for PFL, respectively. Compared with individuals with neither diabetes nor PFL, the adjusted PRs for high psychological distress were: 1.37 (1.17 to 1.60) with diabetes but no PFL, 7.33 (7.00 to 7.67) without diabetes but with severe PFL and 8.89 (8.36 to 9.46) with both diabetes and severe PFL.

Conclusions People with diabetes have a $60 \%$ greater risk of high psychological distress than people without diabetes; a substantial proportion of this elevation is attributable to higher levels of disability with diabetes, especially factoring in measurement error. Psychological distress is strongly related to physical impairment.
Strengths and limitations of this study

- The cohort study used for estimating all-cause mortality risk, the 45 and Up Study, is large and population-based, and the wide range of health data collected from the study population has allowed for multiple variables to be adjusted for.

- The study questionnaire used validated measures of psychological distress and physical functional limitations which ensured the reliability of the data.

- Prevalence ratios of high psychological distress, comparing those with and without diabetes, stratified by level of PFL and need for help with daily tasks were estimated to investigate how much of any observed relationship could be attributed to disability.

- Study limitations include the use of diabetes status determined using self-report. However, a previous study has shown both high sensitivity and specificity for self-reported diabetes in this population, with $98 \%$ of true cases being correctly identified.

- The use of a cross-sectional design meant that neither the causality nor the directions of association could be determined.

\section{INTRODUCTION}

Diabetes is a common chronic condition affecting $9 \%$ of the world population ${ }^{1}$; it is a major cause of morbidity and mortality, and poses a significant challenge for public health systems globally with its increasing prevalence. $^{2}$ The WHO estimated that, in 2014 , there were 422 million people living with diabetes. ${ }^{3}$ People with diabetes not only experience an increased risk of microvascular and macrovascular complications and reduced life expectancy, ${ }^{4}$ but also have reduced physical functioning, social interactions and cognitive status. ${ }^{6-8}$

Psychological distress is approximately two times more prevalent in people with diabetes than people without diabetes. ${ }^{9}{ }^{10}$ Concomitant psychological distress and diabetes have 
been associated with poor glycaemic control, increasing disabilities, loss of productivity and all-cause mortality. ${ }^{11-14}$ Psychological distress is also increased-to an even greater extent-in people living with Physical Functional Limitations (PFLs). ${ }^{15}{ }^{16}$ It is unclear how much of the elevation of psychological distress in people with diabetes is attributable to their higher levels of physical disability. Although many studies have focused on the association between psychological distress and diabetes, ${ }^{9}{ }^{1017}$ there is a lack of reliable evidence on how PFLs affect the association between psychological distress and diabetes. Using a large population-based survey of Australian adults, this study aimed to quantify: (1) the relation of psychological distress to diabetes and (2) the separate and combined associations of diabetes and PFLs with psychological distress, accounting for potential confounding factors.

\section{RESEARCH DESIGN AND METHODS \\ Study population}

The Sax Institute's 45 and Up Study is a population-based cohort study of over 266000 people aged 45 and over in New South Wales, the most populous state of Australia. Individuals joined the study by completing a postal questionnaire distributed between 1 January 2006 and 31 December 2008. ${ }^{18}$ The Department of Human Service (formerly Medicare Australia) mailed questionnaires and information leaflets (available at http://www.45andUp. org.au) to chosen participants in its database. The participants completed the questionnaires. There was a twofold oversampling of people aged 80 years and above and those who lived in rural areas. The overall response rate was $18 \%$, not dissimilar to other longitudinal studies. ${ }^{19}$ Cross-sectional analysis of baseline questionnaire data was used in this study.

\section{Participant involvement}

Participants in the 45 and Up Study receive an annual newsletter informing them of the study progress and projects under way using the study data. The study website is also kept up to date regarding research under way and events. There is no individual feedback to study participants regarding findings or results specific to that individual.

\section{Study measures}

The outcome was psychological distress measured using the Kessler Psychological Distress Scale (K10). The K10 is a validated measure of non-specific symptoms of psychological distress such as feeling 'hopeless' or 'depressed'. ${ }^{20}$ Respondents indicated on a five-point scale, the frequency they experienced each of the symptoms in the past 4 weeks, ranging from 1 'none of the time' to 5 'all of the time'. Scores range from 10 (no distress) to 50 (severe distress); scores were grouped as low $(10$ to $<16)$, moderate (16 to $<22)$, high (22 to $<30$ ) and severe psychological distress $(30-50) .{ }^{21}$ In regression models, high psychological distress was defined as a binary variable (K10 score 22-50).

The main exposure in this study was self-reported diabetes. A participant's diabetes status was identified by his/her answer to the question: 'Has a doctor EVER told you that you have [a number of diseases were listed, including diabetes]?'. The period for which a participant had diabetes was calculated using the participant's reported age at the recruitment into the study and the participant's age when he/she reported being diagnosed with diabetes. This measure has been shown to be $98 \%$ valid, when compared with medical/prescribing records. ${ }^{22}$

PFLs were assessed using the Physical Functioning subscale of the Medical Outcomes Study. ${ }^{23}$ Respondents reported whether they were 'limited a lot', 'a little' or 'not limited at all' when performing a list of 10 activities, ranging from 'vigorous activities' to 'bathing or dressing'. Scores ranged from 0 to 100 , where higher scores represented fewer limitations, and were grouped into four categories: severe (0-59); moderate (60-74), minor (75-99) and no (100) functional limitations. Additionally, participants were asked 'do you regularly need help with daily tasks because of long-term illness or disability?'. Those who answered yes were considered to have severe disabilities.

Covariates considered in this study were age, sex, education, area of residence and country of birth. Participants were categorised into the following 10-year age groups: $45-54,55-64,65-74,75-84$ and $>85$ years. Education was categorised as follows: no educational qualifications, certificate/diploma/school and university education. Area of residence was categorised based on the Accessibility and Remoteness Index of Australia (ARIA+) into major cities, inner regional and more remote areas. Country of birth was categorised into five groups: Australia/New Zealand, Europe/North America, Asia, Africa/Middle-East and other. The other risk factors investigated in the study were annual household income, alcohol consumption per week, smoking status, private health insurance, four categories body mass index (as per the WHO classification, with all types of obesity categorised into one category), ${ }^{24}$ use of language other than English and medical history (cardiovascular diseases, cancer and anxiety/depression).

Participants were included in this study if they had valid data for diabetes status and psychological distress. Of the 266777 participants, 30336 (11\%) who have invalid or missing K-10 scores were excluded from the analysis. There were no missing data for diabetes status. Following exclusions, 236441 participants were eligible to be included in the analysis.

\section{Statistical methods}

The sociodemographic and health behaviour characteristics of the study population were summarised for those with and without diabetes. Patterns of psychological distress in people with and without diabetes were 
summarised using percentages and Fisher's exact confidence intervals (CIs. ${ }^{25}$ Prevalence ratios (PRs) and 95\% CIs for psychological distress, in those with versus without diabetes, were estimated using modified Poisson regression with robust error variance. How much of any observed relationship could be attributed to disability was evaluated by estimating PRs comparing those with and without diabetes, and across categories of diabetes duration, stratified by level of PFL and need for help with daily tasks. Models were initially adjusted for age and sex (model 1) and then further adjusted for education, area of residence, country of birth (model 2). Nested regression models investigated further adjustment for smoking status, alcohol consumption per week in addition to covariates in model 2 (model 3); need for help in daily tasks and PFL in addition to covariates in model 3 (model 4 and model 5, respectively). Missing values in covariates were modelled as a separate category. Tests for trend were performed by modelling the exposure categories as an ordinal variable.

Sensitivity analyses were conducted by adjusting the analyses additionally for other variables such as smoking status, alcohol consumption and disability in the regression model to determine if other factors affected the association between psychological distress and diabetes. A complete case analysis was performed to determine whether the approach to missing data influenced the association. All statistical analyses used a significance level of $5 \%$. Analyses were performed using Stata V.14.

\section{RESULTS}

Of the total study population, $19803(8.4 \%)$ participants reported having diagnosed diabetes. The prevalence of diabetes was higher among: males, older age groups, those born in countries other than Australia, those who spoke languages other than English at home, those with lower education levels, those with lower annual household income, those with no private health insurance, the obese, ex-smokers, those reporting comorbidities such as cardiovascular diseases, cancer (other than skin cancer) and anxiety/depression (table 1). Among those with diabetes, the mean duration of diabetes was 7.7 years; $12 \%, 42 \%$ and $36 \%$ were diagnosed in the past $<2$ years, 2-10 years and $>10$ years, respectively.

The crude prevalence of severe psychological distress (K10 30-50) was twice as high among those with versus without diabetes $(4.1 \%$ vs $2.0 \%$; table 2$)$. High psychological distress (K10 22-50, combining 'severe' and ' $h i g h$ ' distress categories) was greater among participants with versus without diabetes $(11.8 \%$ vs $7.2 \%$, table 2$)$. The pattern of high psychological distress among those with versus without diabetes remained regardless of the duration of diabetes; PRs (95\% CI) were 1.60 (1.44 to 1.79), 1.65 (1.55 to 1.75 ) and 1.84 (1.72 to 1.96) for duration of diabetes $<2$ years, $2-10$ years and $>10$ years, respectively (figure 1).
Table 1 Prevalence of diabetes according to baseline characteristics in the study population

Prevalence of $\mathbf{N}$ with diabetes/ diabetes (\%) total

\begin{tabular}{|c|c|c|}
\hline Overall & 8.4 & (19 803/236 441) \\
\hline \multicolumn{3}{|l|}{ Age group (years) } \\
\hline $45-54$ & 4.5 & (3344/74 969) \\
\hline $55-64$ & 7.9 & $(6292 / 79571)$ \\
\hline $65-74$ & 12.0 & (5947/49 446) \\
\hline $75-84$ & 13.4 & $(3655 / 27$ 195) \\
\hline $85+$ & 10.7 & $(565 / 5260)$ \\
\hline \multicolumn{3}{|l|}{ Sex } \\
\hline Males & 10.3 & (11 431/111 242) \\
\hline Females & 6.7 & (8372/125 199) \\
\hline \multicolumn{3}{|l|}{ Education } \\
\hline No school certificate & 13.4 & (3262/24 334) \\
\hline Certificate/diploma/trade & 8.5 & (12 794/150 411) \\
\hline Tertiary & 5.8 & (3392/58 768) \\
\hline \multicolumn{3}{|l|}{ Annual household income } \\
\hline$<\$ 20000$ & 14.2 & $(6030 / 42578)$ \\
\hline$\$ 20000$ to $<\$ 40000$ & 9.5 & (3933/41 566) \\
\hline$\$ 40000$ to $<\$ 70000$ & 6.5 & (2893/44 232) \\
\hline$\$ 70000+$ & 4.7 & $(2844 / 60811)$ \\
\hline
\end{tabular}

Region of residence

\begin{tabular}{lcl}
\hline Major cities & 8.4 & $(8976 / 106533)$ \\
\hline $\begin{array}{l}\text { Inner regional } \\
\text { More remote }\end{array}$ & 8.4 & $(6997 / 83472)$ \\
\hline $\begin{array}{l}\text { Country of birth } \\
\text { Australia and New }\end{array}$ & 8.3 & $(3829 / 46385)$ \\
$\begin{array}{l}\text { Zealand } \\
\text { Europe/North America }\end{array}$ & 8.0 & $(14572 / 182755)$ \\
$\begin{array}{l}\text { Asia } \\
\text { Africa/Middle East }\end{array}$ & 11.9 & $(3435 / 38145)$ \\
\hline $\begin{array}{l}\text { Other } \\
\text { Private health insurance }\end{array}$ & 11.8 & $(956 / 8055)$ \\
$\quad \begin{array}{l}\text { No private health } \\
\text { insurance }\end{array}$ & 10.6 & $(202 / 1902)$ \\
\hline
\end{tabular}

Health insurance $\quad 7.1 \quad$ (11212/158 282)

Language other than English

at home

$\begin{array}{lrl}\text { No } & 8.0 & (17283 / 214860) \\ \text { Yes } & 11.7 & (2520 / 21579) \\ \text { Smoking status } & & \\ \text { Never smoker } & 8.2 & (9724 / 132917) \\ \text { Past smoker } & 10.0 & (8578 / 85472) \\ \text { Current smoker } & 7.3 & (1432 / 17371)\end{array}$

Alcohol consumption,

drinks/week

\begin{tabular}{rrl}
0 & 12.1 & $(8988 / 74137)$ \\
$1-14$ & 6.5 & $(8068 / 124205)$ \\
\hline & & Continued
\end{tabular}




\section{Table 1 Continued}

\begin{tabular}{|c|c|c|}
\hline & $\begin{array}{l}\text { Prevalence of } \\
\text { diabetes (\%) }\end{array}$ & $\begin{array}{l}\mathrm{N} \text { with diabetes/ } \\
\text { total }\end{array}$ \\
\hline $15+$ & 6.6 & $(2276 / 34583)$ \\
\hline \multicolumn{3}{|l|}{ Body mass index } \\
\hline Underweight & 3.9 & $(98 / 2521)$ \\
\hline Normal weight & 4.5 & $(3596 / 80654)$ \\
\hline Overweight & 7.8 & $(6799 / 87430)$ \\
\hline Obese & 15.7 & (7707/49 121) \\
\hline \multicolumn{3}{|l|}{ Medical history: CVD } \\
\hline No & 4.7 & $(6942 / 146468)$ \\
\hline Yes & 14.3 & (12 861/89 973) \\
\hline \multicolumn{3}{|l|}{ Medical history: cancer } \\
\hline No & 8.0 & (15 956/200 107) \\
\hline Yes & 10.6 & (3847/36 334) \\
\hline \multicolumn{3}{|c|}{ Medical history: anxiety or depression } \\
\hline No & 8.2 & (13 500/165 594) \\
\hline Yes & 9.8 & (3808/38 929) \\
\hline \multicolumn{3}{|c|}{ PFL based on MOS-PF score* } \\
\hline No limitation (100) & 4.4 & $(3278 / 74580)$ \\
\hline Minor limitation (75-99) & 6.8 & $(4270 / 62$ 659) \\
\hline $\begin{array}{l}\text { Moderate limitation (60- } \\
74)\end{array}$ & 10.8 & $(5432 / 50418)$ \\
\hline Severe limitation (0-59) & 18.8 & $(4832 / 25646)$ \\
\hline \multicolumn{3}{|l|}{ Needing help with daily task } \\
\hline No & 7.7 & (16 654/216 069) \\
\hline Yes & 20.9 & $(2338 / 11$ 173) \\
\hline
\end{tabular}

The percentage of missing data (\%): 2928 (1.24\%) for education, $3(<0.01 \%)$ for health insurance, 47254 (20\%) for income, 51 (0.02\%) for area of residence, 1620 (0.69\%) for country of birth, $2(<0.01 \%)$ for language other than English at home, $681(0.29 \%)$ for smoking status, 3516 (1.49\%) for alcohol consumption, 16715 (7.07\%) for body mass index, 1987 (10.03\%) of diabetes duration, 31918 (13.5\%) for anxiety and depression, 23138 (9.79\%) for MOS-PF and 9199 (3.89) need help with daily task.

*MOS-PF, Physical Functioning subscale of the Medical Outcomes Study; PFL, physical functional limitation.
Stratification based on participants' diabetes status and PFLs showed that a substantial portion of this elevation in psychological distress among those with diabetes is attributable to higher levels of PFLs (figure 1). Compared with participants without diabetes or PFLs, the PRs of high psychological distress were 1.35 (95\% CI 1.28 to 1.42 ), 2.84 (95\% CI 2.71 to 2.98 ) and 7.33 (95\% CI 7.00 to 7.67 ) among those without diabetes and with minor, moderate or severe PFLs, respectively. There was a 37\% higher prevalence of high psychological distress in participants with diabetes, but no PFL. In the presence of both diabetes and PFL, the prevalence of high psychological distress was higher compared with participants without diabetes; PRs 1.65 (95\% CI 1.44 to 1.89), 3.52 (95\% CI 3.22 to 3.85 ) and 8.89 (95\% CI 8.36 to 9.46 ) among those with minor, moderate and severe PFLs, respectively (figure 1).

Stratification based on participants' diabetes status and severe disability (needing help with daily tasks) showed that psychological distress is much more strongly related to severe disability than diabetes (figure 1). Compared with participants without diabetes or severe disability, the PR for psychological distress was: 4.80 (95\% CI 4.61 to 4.99) in participants with severe disability only, 1.55 (95\% CI 1.47 to 1.64$)$ in participants with diabetes only and 5.78 (95\% CI 5.44 to 6.14$)$ in those with both diabetes and severe disability.

The PR for high psychological distress among those with versus without diabetes attenuated with further sequential adjustment for sociodemographic characteristics and health behaviours, but remained significantly elevated (PR 1.89 (95\% CI 1.81 to 1.97) in model 1 to 1.58 (1.52 to 1.65 ) in model 3; figure 2). Further adjustment for needing help with daily tasks (model 4, 1.35, 1.29 to 1.40 ) or PFLs (model 5, 1.22, 1.17 to 1.27) further attenuated the PR.

\section{CONCLUSIONS}

In this large population-based study, the prevalence of psychological distress was moderately elevated in people

Table 2 Patterns of psychological distress among people with and without diabetes

\begin{tabular}{llll}
\hline Psychological distress level & N with diabetes/total & $\begin{array}{l}\text { Prevalence in those with } \\
\text { diabetes (\%) }\end{array}$ & $\begin{array}{l}\text { Prevalence in those } \\
\text { without diabetes (\%) }\end{array}$ \\
\hline Low psychological distress & $13946 / 181052$ & $70.4(69.8$ to 71.1$)$ & $77.1(77.0$ to 77.3$)$ \\
Moderate psychological distress & $3518 / 37386$ & $17.8(17.2$ to 18.3) & $15.6(15.5$ to 15.8) \\
High psychological distress & $1533 / 12794$ & $7.7(7.4$ to 8.1$)$ & $5.2(5.1$ to 5.3$)$ \\
Severe psychological distress & $806 / 5209$ & $4.1(3.8$ to 4.4) & $2.0(2.0$ to 2.1) \\
\hline
\end{tabular}

K10 scores for psychological distress were grouped as low $(10$ to $<16)$, moderate $(16$ to $<22)$, high $(22$ to $<30)$ and severe psychological distress (30 to 50 ).

$95 \% \mathrm{Cls}$ are based on Fisher's exact method. 


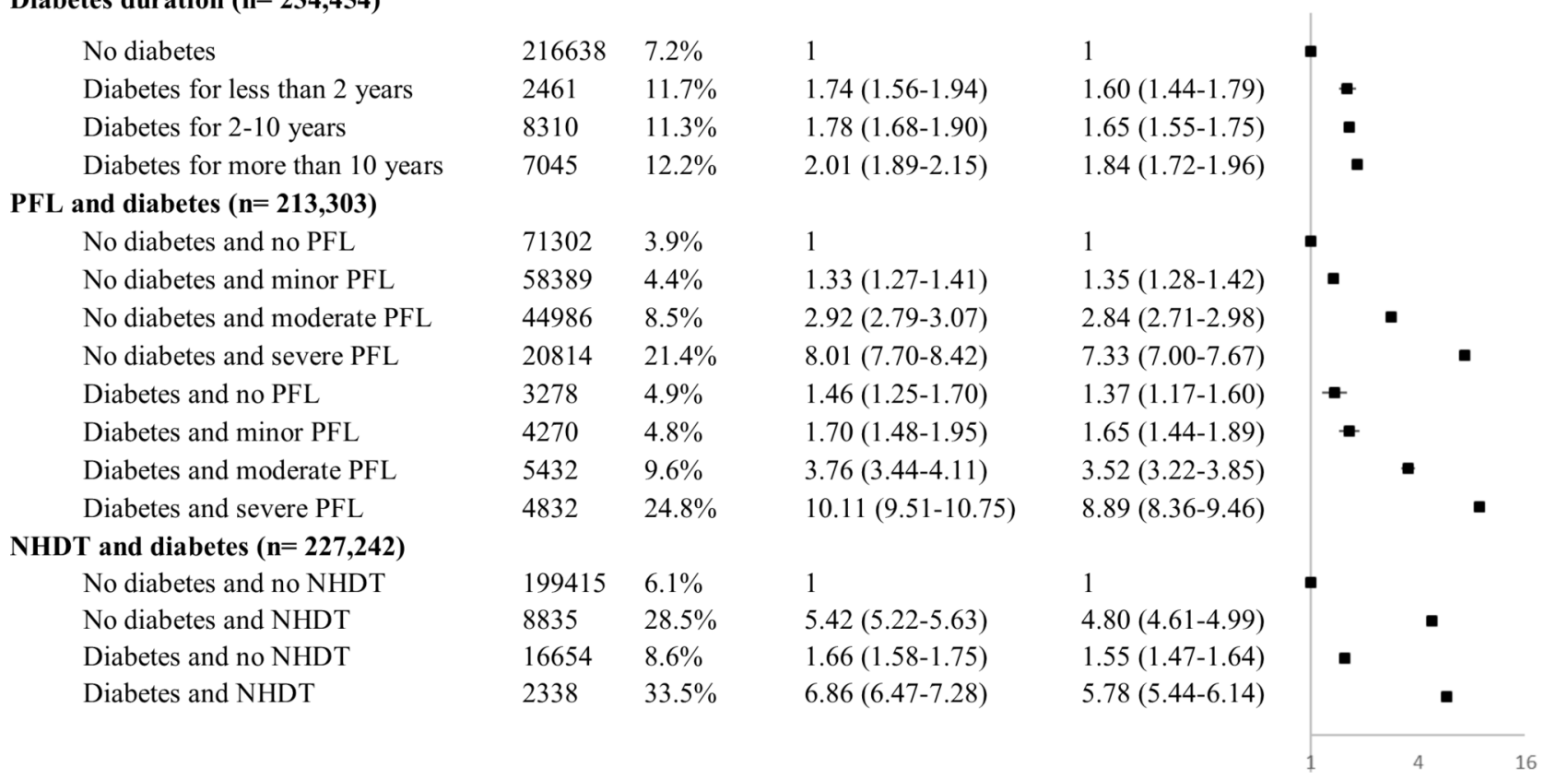

Figure 1 Prevalence ratio of high PD (K10 score 22-50) by duration of diabetes, physical functional limitation and the need for help with daily tasks. ${ }^{1}$ PR of PD adjusted for age and sex. ${ }^{2}$ PR of PD adjusted for age, sex, education, area of residence and country of birth ${ }^{3}$ Test for $p$-trend for each strata showed significant result $(p<0.000)$. NHDT, need for help with daily tasks; PD, psychological distress; PFL, physical functioning limitation; PR, prevalence ratio.

with diabetes versus those without diabetes; a substantial part of this elevation was attributable to higher levels of physical disability. It was shown that the prevalence of psychological distress was seven to eight times higher when participants had severe PFLs regardless of their

\section{PR (95\%CI)}

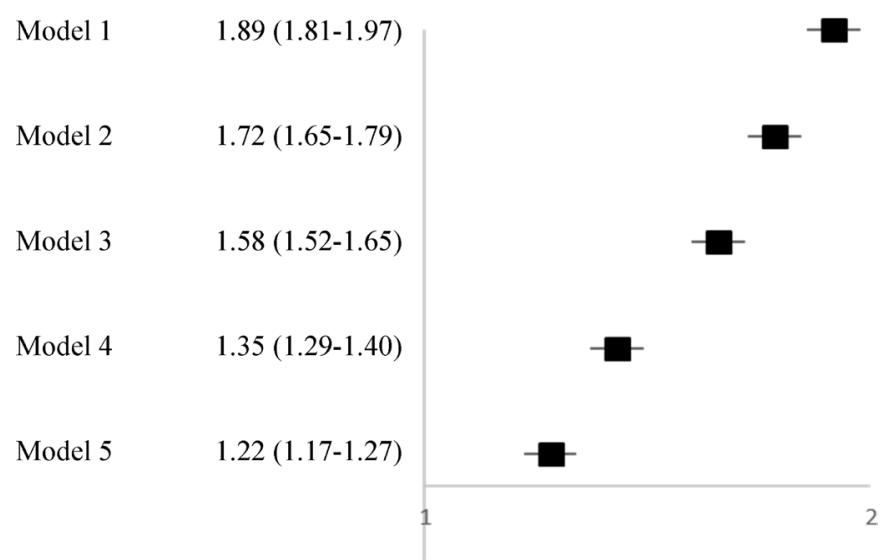

Figure 2 Nested regression models showing the attenuation in prevalence ratio (PR) for high psychological distress in people with versus without diabetes, with further adjustment for confounders. Model 1: adjusted for age and sex; model 2: model 1 is adjusted further for education, area of residence and country of birth; model 3: model 2 is adjusted further for alcohol drink per week and smoking status; model 4: model 3 is adjusted further for need for help with daily tasks; model 5: model 3 is adjusted further for physical functioning limitation. diabetes status. The prevalence of psychological distress was four to six times higher when participants had severe disability regardless of their diabetes status. The excess prevalence of psychological distress attributable to severe PFLs and disability were around 20 times greater and seven times greater, respectively, than those attributable to diabetes alone. Importantly, the point estimate for the relation of diabetes to psychological distress attenuated by $>60 \%$ when further adjusted for PFL; considering that each factor is measured with some degree of error, this means that PFL accounts for the bulk of the observed diabetes-psychological distress relationship.

Previous studies have shown similar mediation by PFL in the association between psychological distress and other disease outcomes, such as heart disease, arthritis and cancer. ${ }^{2627}$ The excess risk of psychological distress attributable to disability is about 40 times greater on average than that attributable to cancer diagnosis, in the absence of disability, among long-term cancer survivors. ${ }^{26}$ Further, the risk of depression in those with arthritis or heart disease was shown to attenuate when activities of daily living and instrumental activities of daily living limitation were considered, demonstrating that physical limitation mediates, to some extent, the association between depression and these conditions. ${ }^{27}$

The findings suggest that functional limitation and disability may mediate the development of psychological distress among those with underlying chronic illness, including diabetes. Depression and physical functioning have been shown to interact with each other in a dynamic 
way, in people with type 2 diabetes; depression at one assessment point might predict poor functioning at the next assessment point which in turn might predict depression at the next assessment point. ${ }^{28}$ Macro and micro vascular diabetes complications such as strokes, peripheral artery diseases, coronary artery diseases, retinopathy and neuropathy may limit people in their physical functioning including self-care. ${ }^{11}{ }^{29}$ Greater disease severity, disability and self-care burden in turn increase psychological distress in people with diabetes. ${ }^{30} 31$

Attenuation in PRs following adjustments for sociodemographic characteristics and health behaviours are consistent with previous findings. Studies conducted in the USA and Australia have showed other risk factors such as sociodemographic factors, health behaviours and diet attenuated the association between psychological distress and diabetes by $30 \%-80 \% .{ }^{9}{ }^{32}$ In this study, the association between diabetes and distress was $54 \%$ lower when adjusted for PFLs and $67 \%$ lower when adjusted for disability.

\section{Limitations and strengths}

This study was conducted using data from the 45 and Up Study (the largest health research study in Australia). The large size of the population of the study and the wide range of health data collected from the study population has allowed for multiple variables to be adjusted for. The study questionnaire used validated measures of psychological distress and PFLs which ensured the reliability of the data.

The findings in this study are subject to some limitations. First, diabetes was determined using self-report. However, a previous study has shown both high sensitivity and specificity for self-reported diabetes in this population, with $98 \%$ of true cases being correctly identified. ${ }^{22}$ Second, the absolute estimates of prevalence in this study may not be representative of the population. However, PRs are based on internal comparisons and remain valid in non-representative cohort studies. ${ }^{334}$ Third, the use of a cross-sectional design meant that neither the causality nor the directions of association could be determined. However, there is growing recognition that the complications associated with type 2 diabetes may translate into functional impairment in older people. ${ }^{35}$ Further, since psychological distress is unlikely to substantively cause diabetes and since the relationship of PFL to distress is well established, having been largely established in other studies, the findings of this study are likely to largely reflect causal associations ${ }^{28}$ between psychological distress and PFL.

In conclusion, the prevalence of high psychological distress is moderately elevated in people with diabetes; a substantial part of this elevation is attributable to physical disability. Other sociodemographic characteristics and health behaviours appear to mediate the association between psychological distress and diabetes. Although causal relationships cannot be established definitively using these data, diabetes management guidelines are likely to benefit from prevention and prompt treatment of diabetes complications which contribute to PFL and disabilities, as well as the identification and effective management of psychological distress.

Acknowledgements This research was completed using data collected through the 45 and Up Study (www.saxinstitute.org.au). The 45 and Up Study is managed by the Sax Institute in collaboration with major partner Cancer Council NSW; and partners: the National Heart Foundation of Australia (NSW Division); NSW Ministry of Health; NSW Government Family \& Community Services-Ageing, Carers and the Disability Council NSW; and the Australian Red Cross Blood Service. The authors thank the many thousands of people participating in the 45 and Up Study.

Contributors GJ and EB designed the study. EE conducted the analyses and drafted the manuscript. GJ provided statistical and methodological support. All authors interpreted the data, and critically revised and approved the final manuscript.

Funding This specific project was supported by a Cardiovascular Disease Network Development Grant from the National Heart Foundation of Australia and a National Health and Medical Research Council of Australia Partnership Grant (GNT1092674). EB is supported by the National Health and Medical Research Council of Australia (1042717).

\section{Competing interests None declared.}

Patient consent Participants provided written consent to take part in the 45 and Up Study

Ethics approval The conduct of the 45 and Up Study was approved by the University of New South Wales Human Research Ethics Committee (HREC). Ethics approval for this study was obtained from the Australian National University Human Ethics Committee (2010/513) and the NSW Population and Health Services Research Ethics Committee (HREC/10/CIPHS/33; CI NSW Study Reference 2010/05/234).

Provenance and peer review Not commissioned; externally peer reviewed.

Data sharing statement Information about data access and governance policies is available at https://www.saxinstitute.org.au/our-work/45-up-study/for-researchers/

Open access This is an open access article distributed in accordance with the Creative Commons Attribution Non Commercial (CC BY-NC 4.0) license, which permits others to distribute, remix, adapt, build upon this work non-commercially, and license their derivative works on different terms, provided the original work is properly cited, appropriate credit is given, any changes made indicated, and the use is non-commercial. See: http://creativecommons.org/licenses/by-nc/4.0/.

\section{REFERENCES}

1. Federation ID. IDF Diabetes Atlas. Seventh Edition, 2015.

2. Zimmet PZ, Magliano DJ, Herman WH, et al. Diabetes: a 21st century challenge. Lancet Diabetes Endocrinol 2014;2:56-64.

3. World Health Organization. Global report on diabetes. Geneva: WHO, 2016.

4. Andrade FC. Measuring the impact of diabetes on life expectancy and disability-free life expectancy among older adults in Mexico. J Gerontol B Psychol Sci Soc Sci 2010;65B:381-9.

5. Shiu AT, Choi KC, Lee DT, et al. Application of a health-related quality of life conceptual model in community-dwelling older Chinese people with diabetes to understand the relationships among clinical and psychological outcomes. J Diabetes Investig 2014;5:677-86.

6. Egede LE, Dismuke CE. Serious psychological distress and diabetes: a review of the literature. Curr Psychiatry Rep 2012;14:15-22.

7. Australian Institute of Health and Welfare. Diabetes and disability: impairments, activity limitations, participation restrictions and comorbidities. Canberra: AlHW, 2013.

8. Rosso AL, Taylor JA, Tabb LP, et al. Mobility, disability, and social engagement in older adults. J Aging Health 2013;25:617-37.

9. Li C, Ford ES, Zhao G, et al. Association between diagnosed diabetes and serious psychological distress among U.S. adults: the Behavioral Risk Factor Surveillance System, 2007. Int J Public Health 2009;54 Suppl 1:43-51.

10. Shin JK, Chiu YL, Choi S, et al. Serious psychological distress, health risk behaviors, and diabetes care among adults with type 2 diabetes: the California Health Interview Survey 2007. Diabetes Res Clin Pract 2012;95:406-14. 
11. Dalsgaard EM, Vestergaard M, Skriver MV, et al. Psychological distress, cardiovascular complications and mortality among people with screen-detected type 2 diabetes: follow-up of the ADDITIONDenmark trial. Diabetologia 2014;57:710-7.

12. Schmitz N, Wang J, Lesage A, et al. Psychological distress and short-term disability in people with diabetes: results from the Canadian Community Health Survey. J Psychosom Res 2008;65:165-72.

13. Australian Institute of Health and Welfare. Diabetes and poor mental health and wellbeing: an exploratory analysis. Canberra: AlHW, 2011.

14. Ascher-Svanum H, Zagar A, Jiang D, et al. Associations between glycemic control, depressed mood, clinical depression, and diabetes distress before and after insulin initiation: an exploratory, post hoc analysis. Diabetes Ther 2015;6:303-16.

15. Banks E, Byles JE, Gibson RE, et al. Is psychological distress in people living with cancer related to the fact of diagnosis, current treatment or level of disability? Findings from a large Australian study. Med J Aust 2010;193:S62-7.

16. Prince M, Patel V, Saxena S, et al. No health without mental health. The Lancet 2007;370:859-77.

17. Lloyd C, Smith J, Weinger K. Stress and diabetes: a review of the links. Diabetes Spectrum 2005;18:121-7.

18. Banks E, Redman S, Jorm L, et al. Cohort profile: the 45 and Up Study. Int J Epidemiol 2008;37:941-7.

19. Edwards S, Fraser S, Rauch J, et al. CHIS 2007 Methodology Report Series, 2009.

20. Kessler RC, Andrews G, Colpe LJ, et al. Short screening scales to monitor population prevalences and trends in non-specific psychological distress. Psychol Med 2002;32:959-76.

21. Andrews G, Slade T. Interpreting scores on the Kessler Psychologica Distress Scale (K10). Aust N Z J Public Health 2001;25:494-7.

22. Comino EJ, Tran DT, Haas M, et al. Validating self-report of diabetes use by participants in the 45 and Up Study: a record linkage study. BMC Health Serv Res 2013;13:481.

23. Stewart A, Kamberg C. Measuring functioning and well-being: the Medical Outcomes Study Approach. Durham, North Carolina: Duke University Press, 1992.
24. World Health Organization. Body Mass Index-BMI. 2016 http://www. euro.who.int/en/health-topics/disease-prevention/nutrition/a-healthylifestyle/body-mass-index-bmi (accessed 25 Apr 2016).

25. Armitage P, Berry PJ, Matthews JNS. Statistical methods in medical research. 4th ed. Oxford: Blackwell Publishing, 2002.

26. Banks E, Byles JE, Gibson RE, et al. Is psychological distress in people living with cancer related to the fact of diagnosis, current treatment or level of disability? Findings from a large Australian study. Med J Aust 2010;193:S62.

27. Dunlop DD, Lyons JS, Manheim LM, et al. Arthritis and heart disease as risk factors for major depression: the role of functional limitation. Med Care 2004;42:502-11.

28. Schmitz N, Gariépy G, Smith KJ, et al. Longitudinal relationships between depression and functioning in people with type 2 diabetes. Ann Behav Med 2014:47:172-9.

29. Boini S, Erpelding ML, Fagot-Campagna A, et al. Factors associated with psychological and behavioral functioning in people with type 2 diabetes living in France. Health Qual Life Outcomes 2010;8:124.

30. Delahanty LM, Grant RW, Wittenberg E, et al. Association of diabetes-related emotional distress with diabetes treatment in primary care patients with Type 2 diabetes. Diabet Med 2007;24:48-54.

31. Gomes MB, Negrato CA; Retirement due to disabilities in patients with type 1 diabetes a nationwide multicenter survey in Brazil. BMC Public Health 2015;15:486.

32. Byles JE, Robinson I, Banks E, et al. Psychological distress and comorbid physical conditions: disease or disability? Depress Anxiety 2014;31:524-32.

33. Mealing NM, Banks E, Jorm LR, et al. Investigation of relative risk estimates from studies of the same population with contrasting response rates and designs. BMC Med Res Methodol 2010;10:26.

34. Rothman KJ, Gallacher JE, Hatch EE. Why representativeness should be avoided. Int J Epidemiol 2013;42:1012-4.

35. De Rekeneire N, Resnick HE, Schwartz AV, et al. Diabetes is associated with subclinical functional limitation in nondisabled older individuals: the Health, Aging, and Body Composition study. Diabetes Care 2003;26:3257-63. 